\section{Detektivarbeit im Pflanzenreich}

D er Trend zur Selbstmedikation mit pflanzlichen Arzneimitteln hält an. Obwohl bis auf wenige Ausnahmen Phytopharmaka nicht mehr zu Lasten der gesetzlichen Krankenkassen verordnet werden dürfen, liegt allein in Deutschland der Jahresumsatz bei über einer Milliarde Euro - mit steigender Tendenz. Diese Beliebtheit verdanken pflanzliche Arzneimittel der zumindest in Laienkreisen weit verbreiteten Auffassung, dass „Naturprodukte“ gar keine Nebenwirkungen haben. Doch es gilt auch hier: Was wirkt, hat auch Nebenwirkungen - und was keine Nebenwirkungen hat, wirkt wohl auch nicht.

Das hier vorgestellte, kürzlich erschienene Buch stellt nicht nur mögliche Nebenwirkungen, sondern auch mögliche Wirkungen von pflanzlichen Arzneimitteln dar. So erfährt man, dass beispielsweise Teebaumöl nachgewiesenermaßen über antiseptische, bakterizide und fungizide Wirkungen verfügt. Aber, wie schon gesagt, was wirkt, hat auch Nebenwirkungen, und so kann es durch eine systemische Überdosierung zu Übelkeit, Durchfall oder neurologischen Ausfallserscheinungen kommen. Frisches Teebaumöl wirkt nur als schwaches Kontaktallergen, aber welcher Patient hat zu Hause schon ein analytisches Labor und kann die Entstehung potenter Allergene durch Oxidation überprüfen?

So wird systematisch und mit vielen Detailinformationen eine Auswahl von 60 Pflanzen abgehandelt. Dabei onen vor. wird nach potenziellen Auslösern allergischer Kontaktekzeme und allergischer Soforttypreaktionen unterteilt. Nicht wenige Pflanzen kommen in beiden $\mathrm{Ru}$ briken vor. Pharmakologische Wirkungen, Anwendungsmöglichkeiten, Effekte auf die Haut und allergische Reaktionen nach arzneilicher Zubereitung werden Pflanze für Pflanze abgehandelt. Zur Beruhigung: Keineswegs ist jede Pflanze allergologisch bedeutsam, bei vielen Heilpflanzen liegen noch keine publizierten Fälle allergischer Reakti-

Dass sich die Autoren alle Mühe gegeben haben, auch noch die abseitigsten und verstecktesten Publikationen nach Fallberichten oder Untersuchungen zu allergischen Reaktionen auf pflanzliche Arzneimittel zu durchforsten, lässt sich aus dem Umfang der ausgewerteten Literatur erahnen: Fast 1.300 Zitate sind aufgelistet. Sogar originalsprachige Literatur aus Japan, China und Osteuropa wurde berücksichtigt!

Das Ergebnis dieser intensiven Recherche ist aber keineswegs so trocken und langweilig, wie man angesichts der Fülle an ausgewerteter Literatur befürchten mag, sondern das Buch liest sich streckenweise so vergnüglich, dass es sich nahezu als Unterhaltungslektüre eignet. Einige Beispiele aus der Praxis der Selbstmedikation: Eine Patientin versuchte, ein Lipom mit Lorbeeröleinreibungen zu beseitigen, was zwar nicht gelang, aber eine allergische Kontakt- dermatitis auslöste. Oder: Knoblauchtabletten sollten gegen Hyperlipidämie helfen, führten aber zu einem massiven Handekzem im Sinne eines hämatogen allergischen Kontaktekzems. Nicht erwähnt wird leider, ob sich die Fettstoffwechselstörung besserte. Und so geht es in einem fort das ganze Buch hindurch.

Das Buch gibt dem Detektiv, der wohl in jedem Allergologen stecken muss, viele Anregungen. Das Buch enthält weiter - allerdings kleinformatige - farbige Abbildungen der Pflanzen und ein umfangreiches Literaturverzeichnis. $\mathrm{Zu}$ bemängeln ist bei diesem ansonsten rundum empfehlenswerten Buch allenfalls eine unerfreuliche Diskrepanz zwischen Form und Inhalt. Das in Ringbroschur gebundene Paperback dürfte bei häufigerer Benutzung vermutlich bald in seine Einzelteile zerfallen, was bei einem Preis von immerhin fast 90 Euro nicht wirklich nachzuvollziehen ist.

Priv.-Doz. Dr. Franziska Ruëff, München

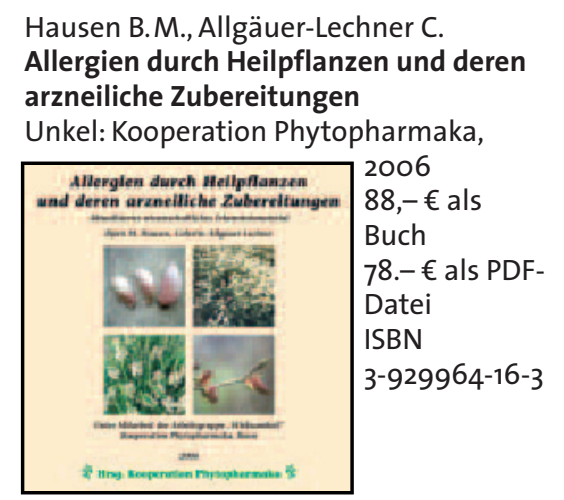

ähnlich erfolgreich sein wie schon die vorherigen.

Dr. Ulrich Mutschler, Hildesheim

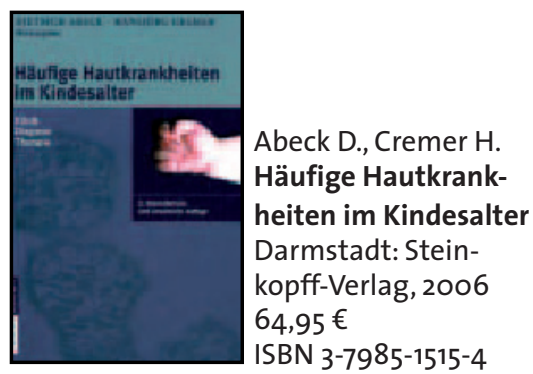

ISBN 3-7985-1515-4 anderen Kapitel der zweiten Auflage wurden überarbeitet und weitere Erkrankungen hinzugefügt. Das Buch besticht wieder durch hervorragende $\mathrm{Ab}$ bildungen, klare Differenzialdiagnosen und seinen praxisrelevanten Zugang. Es deckt so über $95 \%$ der alltäglichen Probleme des Kinderarztes mit hautkranken Kindern ab. Auch die neue Auflage wird mit diesem klaren Konzept sicher handlung des atopischen Ekzems eine wichtige Rolle spielen werden. Auch die ie letzte Auflage dieses kompakten und bewährten Praxisbuches liegt schon fünf Jahre zurück. In der Zwischenzeit sind in der pädiatrischen Dermatologie wieder neue Therapiemöglichkeiten hinzugekommen, darunter vor allem die topischen Calcineurin- 